\title{
Novel Rate-Control Algorithm Based on TM5 Framework
}

\author{
Zhongjie ZHU ${ }^{1,2}$, Yongqiang BAI ${ }^{1,2}$, Zhiyong DUAN ${ }^{1,2}$, Feng LIANG ${ }^{1}$ \\ ${ }^{1}$ Ningbo Key Laboratory of DSP, Zhejiang Wanli University, Ningbo, China \\ ${ }^{2}$ Physical Engineering College, Zhengzhou University, Zhengzhou, China \\ E-mail: zhongjiezhu@hotmail.com \\ Received April 9, 2009; revised April 25, 2009; accepted May 30, 2009
}

\begin{abstract}
Rate control is a key technology in the fields of video coding and transmission, and it has attracted a great attention and has been studied extensively. The TM5 framework of MPEG-2 is a classical rate control algorithm and has being widely used. However, it has some underlying drawbacks during practical applications such as the poor rate control precision and high computational complexity. Hence, in this paper, a novel rate-control algorithm based on the TM5 framework is proposed. The drawback of the target bit allocation method of the original TM5 algorithm is firstly analyzed and improved. Then, a new rate-distortion model is incorporated into the rate control algorithm to implement rate prediction to enhance the rate-control precision. Meanwhile, the macro-block (MB) level rate control is adapted to be frame level to reduce the computational complexity. Experiments are conducted and some results are given. Compared with the original TM5 algorithm, the improved novel algorithm not only can enhance the rate-control precision but also can reduce the complexity and the fluctuation of decoded image quality.
\end{abstract}

Keywords: Rate Control, TM5, Target Bit Allocation, Rate-Distortion Model

\section{Introduction}

Rate control is a key technology in the fields of video coding and transmission [1,2]. With the rapid progress of video coding technology and explosion of video applications, it has attracted a great attention and has been studied extensively. The main objective of rate control is to optimally allocate available bits within video sequences to minimize visual distortion under the bit rate constraint. For a rate control algorithm, the rate-distortion performance and the computational complexity are two main issues that should be addressed. Although rate control is a normative part in video coding standards, almost all the main existing video coding standards have proposed their own recommendations on rate control over the last few years such as the TM5 algorithm of MPEG-2, the VM8 algorithm of MPEG-4, the TMN8 algorithm of H.263 and the F086/G012 of H.264 [3,4].

As mentioned above, the rate control is an informative part in video coding standard, which means that this part is still open for research. It leaves the flexibility for designers to develop suitable scheme for specific applications. Hence, this topic is still being studied extensively. $\mathrm{Xu}$ et al have proposed a novel Dynamic Video Rate
Control (DVRC) technique which can enable adaptive video delivery over the Internet [5]. But its performance in heterogeneous network environments should be further enhanced. Papadimitriou et al have proposed a novel rate control algorithm when hierarchical B-picture coding is used in H.264/AVC, where significant PSNR gains as well as accurate rate control precision can be achieved [6]. However, the computational load is high. For more other related works, the readers are referred to [2].

It is noted that, the TM5 rate control algorithm of MPEG-2 has obtained great attention and has been widely used. It implements rate control in macro-block level and mainly consists of three steps: target bit allocation, rate control and adaptive quantization. However, it also has drawbacks including poor rate-control precision and not low computational complex due to its macrolevel quantification $[7,8]$. In this paper, a novel ratecontrol algorithm based on TM5 framework is proposed. The target bit-allocation of TM5 is improved and a new rate-distortion model is incorporated to implement rate prediction to enhance the rate-control precision. Meanwhile, the macro-block level rate control is adapted to frame level to reduce the computational complexity.

The remaining of the paper is organized as follows: 
The TM5 rate control algorithm is briefly reviewed in Section 2; the target bit allocation is analyzed and an improved rate-distortion model is introduced in Section 3; the proposed novel algorithm is introduced in Section 4; Section 5 shows the experimental results to evaluate our work; Section 6 concludes the paper.

\section{TM5 Rate Control Algorithm}

The TM5 rate control algorithm has been designed for MPEG-2 standard. It mainly consists of the following three steps:

\subsection{Target Bit Allocation}

The target number of bits for the next picture depends on picture-type and "universal" weighting factors. The target number of bits for different type of frames $\left(T_{I}, T_{P}\right.$, $T_{B}$ ) are calculated by [9]:

$$
\begin{gathered}
T_{I}=\frac{R}{1+\frac{N_{P} X_{P}}{K_{P} X_{I}}+\frac{N_{B} X_{B}}{K_{B} X_{I}}} \\
T_{P}=\frac{R}{N_{P}+\frac{N_{B} K_{P} X_{B}}{K_{B} X_{P}}} \\
T_{B}=\frac{R}{N_{B}+\frac{N_{P} K_{B} X_{B}}{K_{P} X_{B}}}
\end{gathered}
$$

where $R$ is the remaining number of bits assigned to GOP, $N_{P}, N_{B}$ are the number of P-pictures and B-pictures remaining in the current GOP, $K_{P}, K_{B}$ are universal constants depending on the quantization matrices. In most cases, $K_{P}=1.0$ and $K_{B}=1.4$.

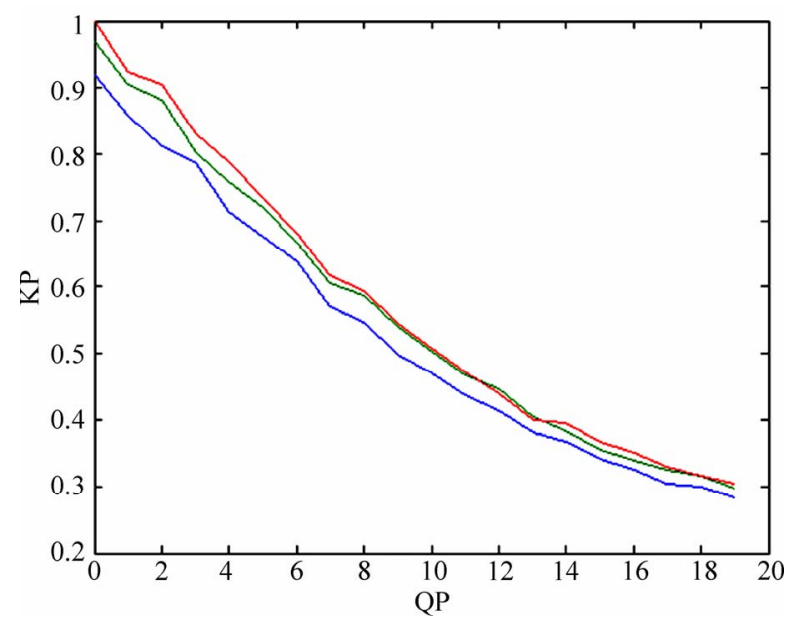

(a) $K_{\mathrm{P}}-\mathrm{QP}$ curves of three $\mathrm{P}$-frames

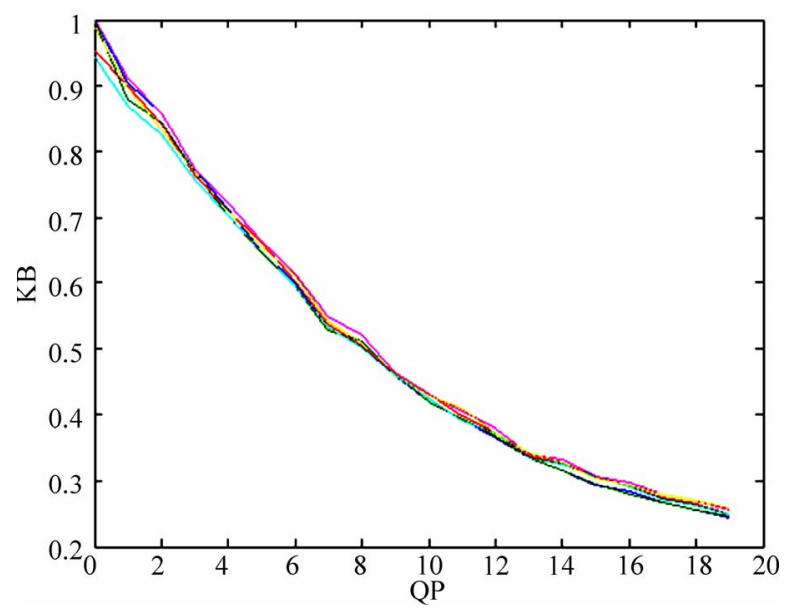

(b) $K_{B}-Q P$ curves of the six $B$-frames

Figure 1. The $\left(K_{P}, K_{B}\right)$-QP curves of Alex sequence from one GOP.

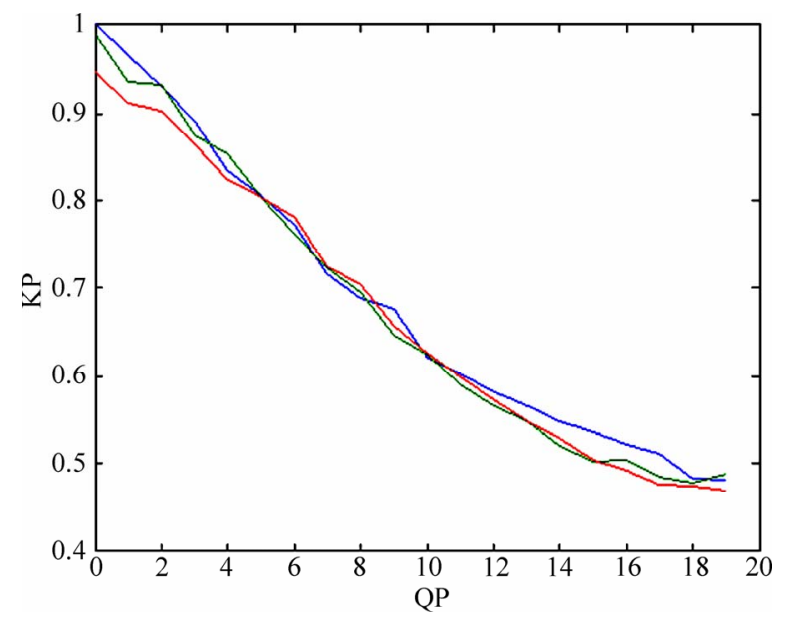

(a) $K_{\mathrm{P}}-\mathrm{QP}$ curves of the three $\mathrm{P}$-frames

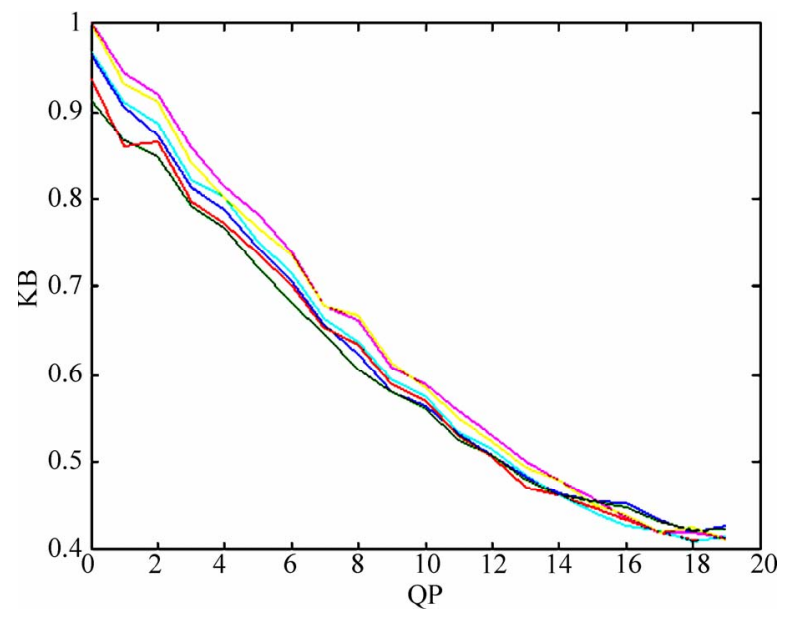

(b) $K_{B}-Q P$ curves of the six $B$-frames

Figure 2. The $\left(K_{P}, K_{B}\right)-Q P$ curves of the Claire sequence from one GOP. 


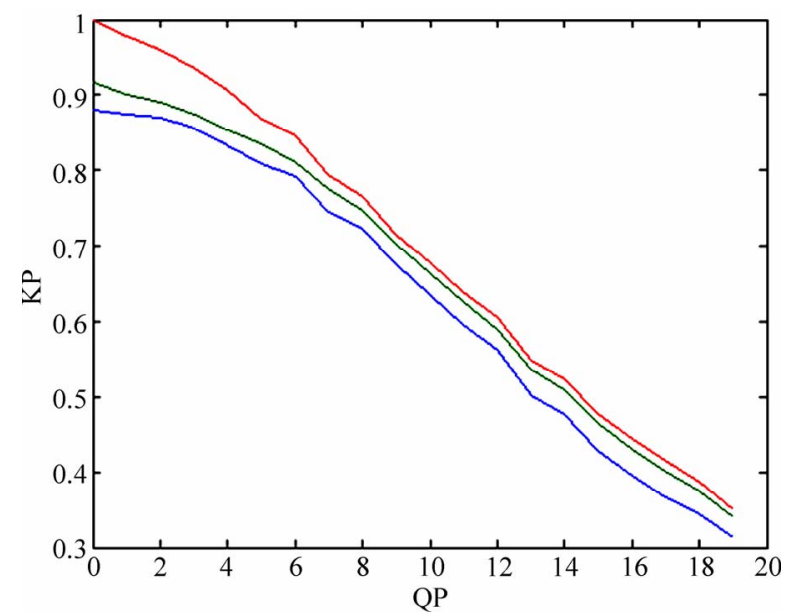

(a) $K_{\mathrm{P}}-\mathrm{QP}$ curves of the three $\mathrm{P}$-frames

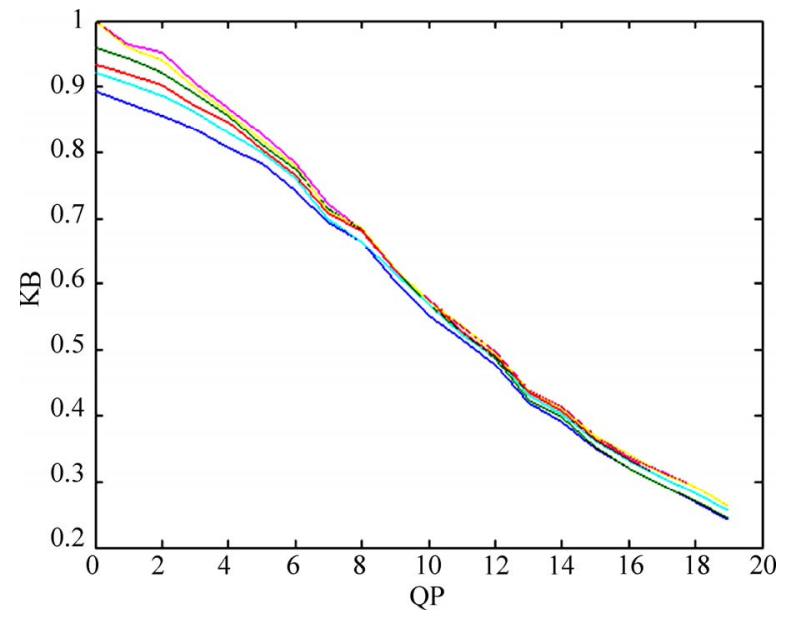

(b) $K_{\mathrm{B}}-\mathrm{QP}$ curves of the six $\mathrm{B}$-frames

Figure 3. The $\left(K_{P}, K_{B}\right)-Q P$ curves of the Train sequence from one GOP.

\subsection{Rate Control}

The reference value of the quantization parameter for each macro-block (MB) $Q_{j}$ is set as follows:

$$
Q_{j}=\left(\frac{d_{j} \times 31}{r}\right)
$$

where $r$ is the reaction parameter and given by

$$
r=2 \frac{R}{f}
$$

where $R$ denotes the bit rate, $f$ denotes the frame rate.

\subsection{Adaptive Quantization}

The final quantification parameter mquant $_{j}$ for the $j$ th
Macro-block $M B_{j}$ is given by

$$
\text { mquant }_{j}=Q_{j} \times N_{-} a c t_{j}
$$

where $N_{-} a c t_{j}$ is the normalized spatial activity measured for $M B_{j}$.

\section{Improved Target Bit Allocation and Rate Distortion Model}

\subsection{Improved Target Bit Allocation}

From Formulas (1), (2) and (3), it can be seen that $K_{P}$, $K_{B}$ are universal constants depending on the quantification matrix, which can be viewed as the ratio of number of bits of I frame to that of P frame or B frame, respectively, that is

$$
K_{P}=\frac{R_{I}}{R_{P}}, K_{B}=\frac{R_{I}}{R_{B}}
$$

where $R_{I}, R_{P}, R_{B}$ denote the number of bits of I, P, $B$ frames respectively.

In practical video coding applications, $K_{P}$ and $K_{B}$ are not constant, they are usually related to the quantification parameter of I-frame. Hence, rate control error and video quality fluctuation may increase if $K_{P}$ and $K_{B}$ keep constant during the whole encoding process.

To investigate the actual relations between $K_{P}$, $K_{B}$ quantification parameter of I-frame, experiments are conducted. Some standard test sequences, including Alex, Claire, and Train, are used to implement coding experiments to investigate this phenomenon. The GOP structure is set to IBBPBBPBBP [10]. Some experimental results are given in Figures 1-3 where the values of $K_{P}$ and $K_{B}$ are normalized and the actual $K_{P}-Q P$ curves of three P-frames and the $K_{B}-Q P$ curves of six $B$-frames from the same GOP are given. From the experimental results, it can be observed that the decrease of $K_{P}$ or $K_{B}$ is approximately linear to the increment of $Q P$ of I-frame.

Hence, the $K_{P}-Q P$ or $K_{B}-Q P$ relations can be approximately modeled as

$$
K_{i}=a \times Q P+b \quad i \in\{P, B\}
$$

where $K_{i}$ denotes the frame-level bit-allocation coefficient of $\mathrm{P}$ or B frames, $Q P$ is the quantification parameter of I frame, $a$ and $b$ are model parameters. 


\subsection{Novel Rate-Distortion Model}

R-D models have been introduced since MPEG-4 and H.263. These models are helpful in rate control since they can provide sufficient information for determining quantification parameters. Once the target bit rate for the current encoding frame is acquired, the quantization parameter can be determined through the R-Q models. In order to improve the precision of the traditional quadratic R-D model, based on empirical observations and lots of experiments, we improved the quadric R-Q model and proposed a novel one [11]:

$$
R=\frac{a}{\sqrt{Q}}+\frac{b}{Q^{2}}+c
$$

where a, b, c are model parameters which can be calculated by linear regression method explained as follows. Define

$$
x_{1}(Q)=\frac{1}{Q^{2}}, \quad x_{2}(Q)=\frac{1}{\sqrt{Q}}
$$

Suppose $\left(x_{11}, x_{21}, R_{1}\right),\left(x_{12}, x_{22}, R_{2}\right), \ldots,\left(x_{1 n}, x_{2 n}, R_{n}\right)$ are existing samples, and define

$$
M=\left(\begin{array}{ccc}
1 & x_{11} & x_{21} \\
1 & x_{12} & x_{22} \\
\vdots & \vdots & \vdots \\
1 & x_{1 n} & x_{2 n}
\end{array}\right), R=\left(\begin{array}{c}
R_{1} \\
R_{2} \\
\vdots \\
R_{n 1}
\end{array}\right), C=\left(\begin{array}{c}
c \\
b \\
a
\end{array}\right)
$$

Then based on linear regression method, the parameters can be estimated:

$$
C=\left(M^{T} M\right)^{-1} M^{T} R,
$$

where $M^{T}$ is the transpose of $M$ and $\left(M^{T} M\right)^{-1}$ is the inverse matrix of $M^{T} M$.

\section{Proposed Algorithm}

Based on the above observations, the traditional TM5 rate control framework is adapted and a new rate control algorithm is proposed. The target bit-allocation is improved and a new rate-distortion model is incorporated to implement rate prediction to enhance the rate-control precision. Meanwhile, the macro-block level rate control is adapted to frame level to reduce the computational complexity. The key steps of the rate control are briefly introduced as follows:

\subsection{Target Bits Calculation}

Suppose $N_{G}$ is the length of GOP, $f_{r}$ is the frame- rate, $b_{r}$ is the bit-rate, the initial target number of bits for a GOP is calculated as:

$$
T(0)=\frac{b_{r}}{2 f_{r}} N_{G}-\left(B_{0}-B_{c}(0)\right)
$$

where $B_{0}$ denotes the initial value of virtual buffer, $B_{c}(j)$ denotes the occupancy of virtual buffer after encoding the $j$ th frame, $B_{c}(0)$ denotes the occupancy of virtual buffer after encoding the former GOP, $T(j)$ denotes the remaining bits available for the current GOP after encoding the $j$ th frame. After encoding one frame, $T(j)$ is updated as:

$$
T(j)=T(j-1)-A(j-1)
$$

where $A(j)$ is the number of bits generated by encoding the $j$ th frame.

For frame-level bit-allocation, it is very important to properly select $K_{P}$ and $K_{B}$. The bigger the values of $K_{P}$ and $K_{B}$ are, the smaller the distortion of encoded I frame will be. However, if $K_{P}$ and $K_{B}$ are set too large values, not only will the stream fluctuation increase but also video quality will decrease. Hence, in our algorithm, $K_{P}$ and $K_{B}$ are selected according to Equation (8).

\subsection{Frame-Level Rate Control}

After the target number of bits for each frame is estimated, the quantification parameter can be calculated through the following rate-distortion model:

$$
\frac{R-H}{X}=\frac{a}{\sqrt{Q}}+\frac{b}{Q^{2}}+c
$$

where $a, b, c$ are model parameters, $H$ is the head information. The complexity $X$ of current frame is expressed by $S A D$ predicted from that of the former frame, which is calculated as:

$$
S A D=\sum_{(x, y)} a b s(f(x, y)-\overline{f(x, y)})
$$

\subsection{Model Update}

When finishing encoding one frame, the $S A D$ model and the $R-Q$ model are updated until the whole video sequence is encoded.

\section{Experiment Results}

In order to evaluate the performance of the proposed rate control algorithm, we conduct theoretical analysis as 


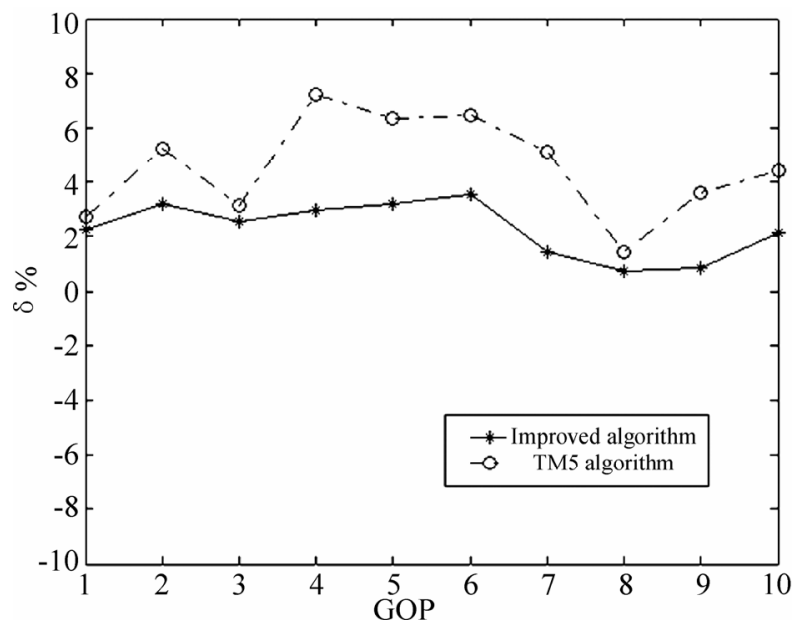

(a) 160Kbps

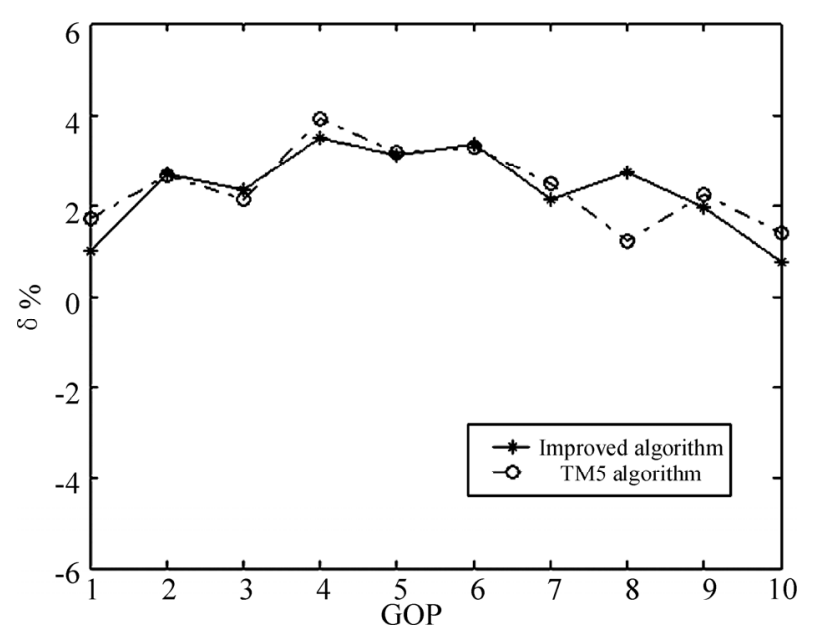

(b) 150Kbps

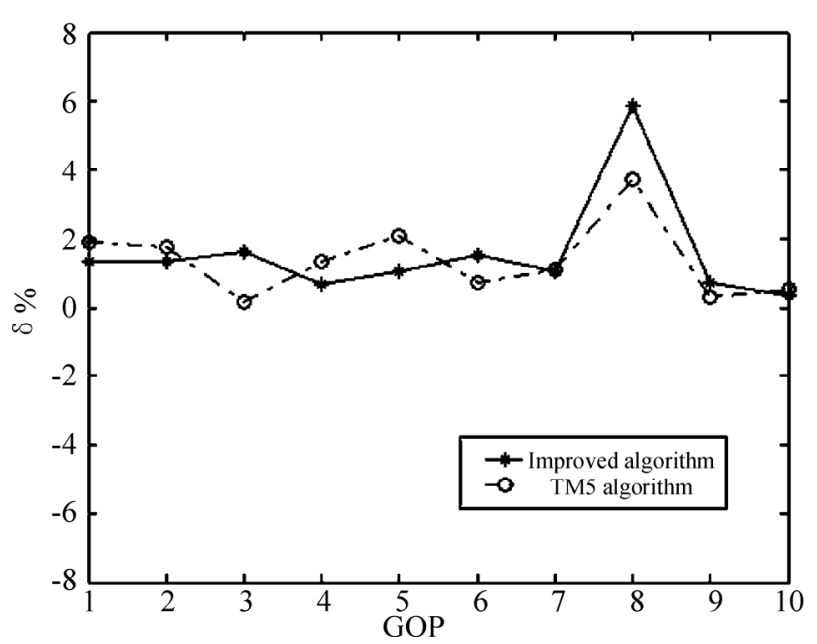

(c) 130Kbps

Figure 4. Rate-control precisions of the Alex sequence at different bit rates.

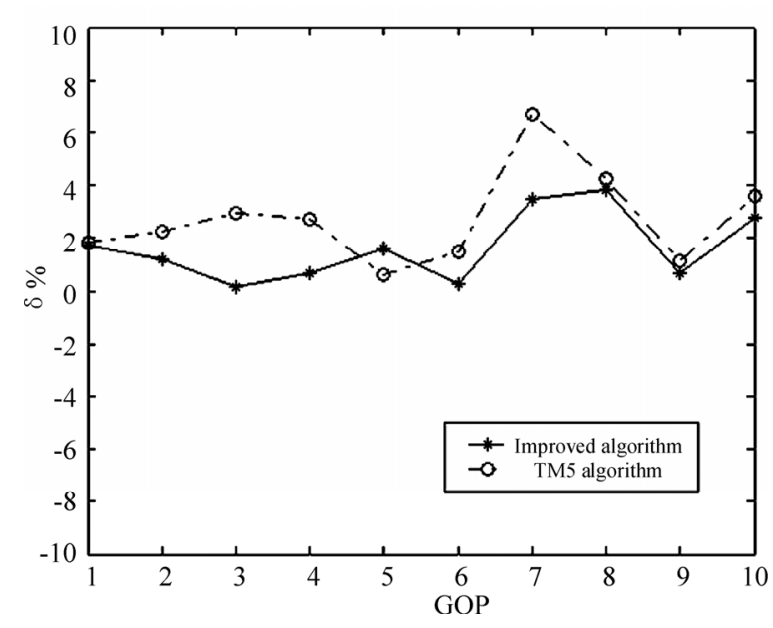

(a) $1.2 \mathrm{Mbps}$

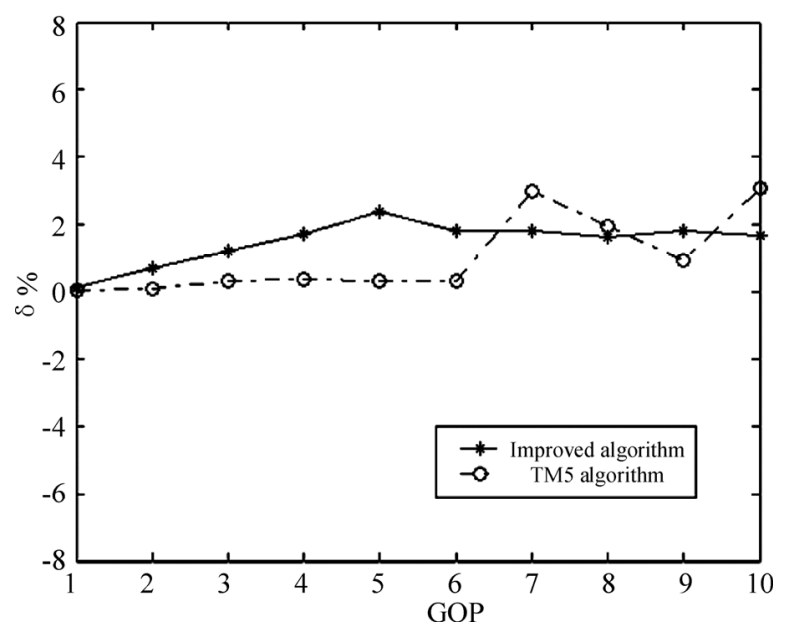

(b) 1.0Mbps

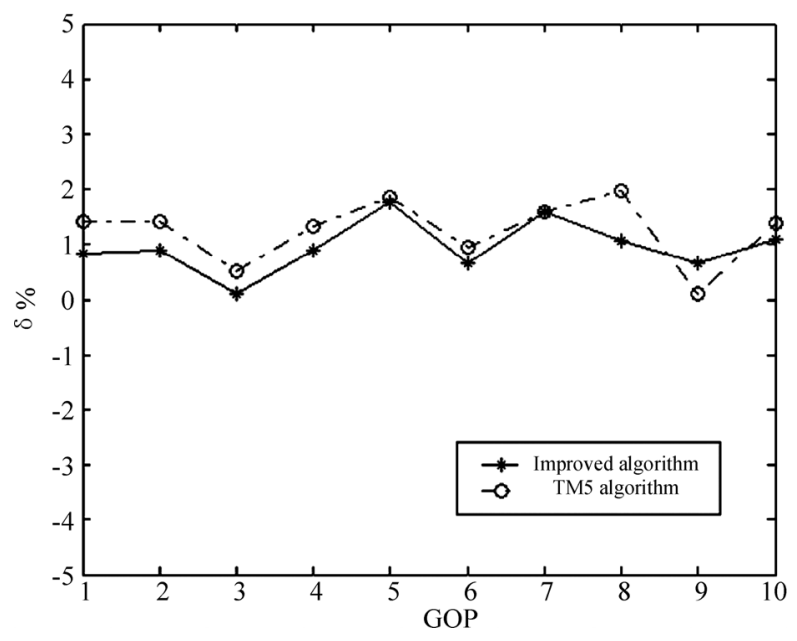

(c) $0.6 \mathrm{Mbps}$

Figure 5. Rate-control precisions of the Train sequence at different bit rates. 


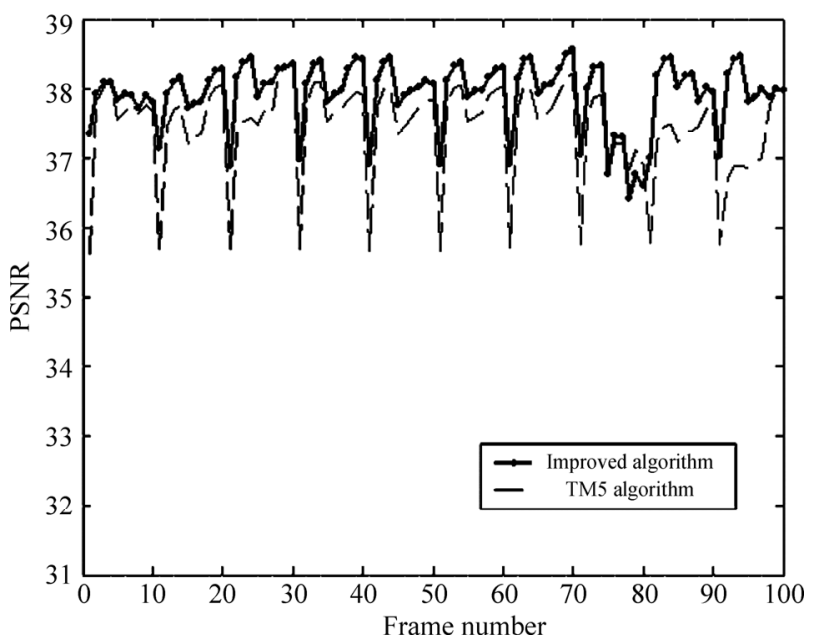

(a) 160Kbps

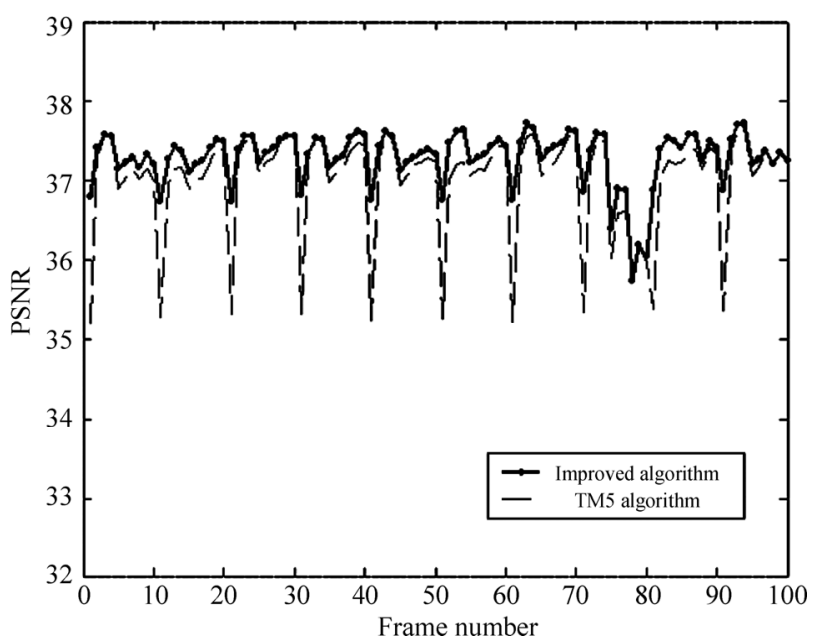

(b) 150Kbps

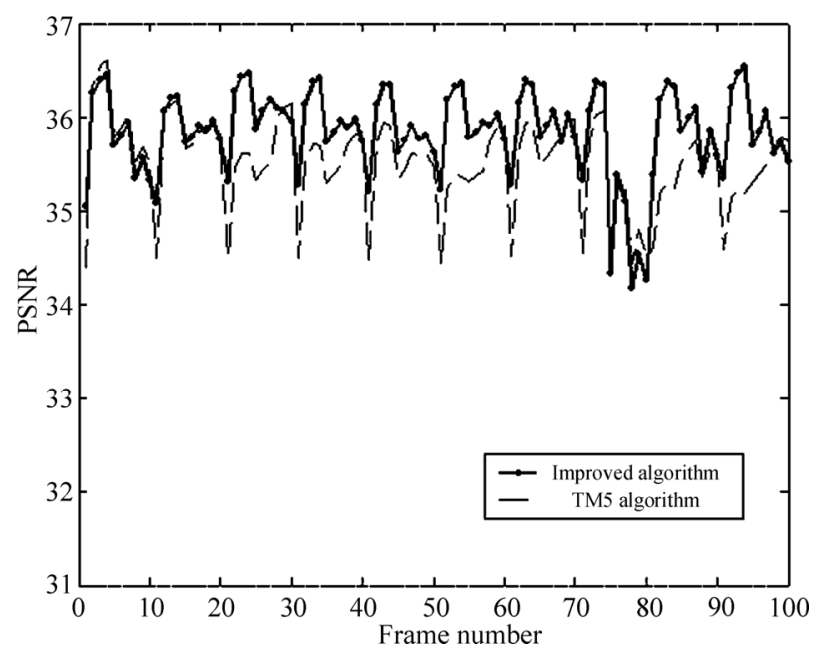

(c) 130Kbps

Figure 6. Image qualities of the Alex sequence at different bit rates.

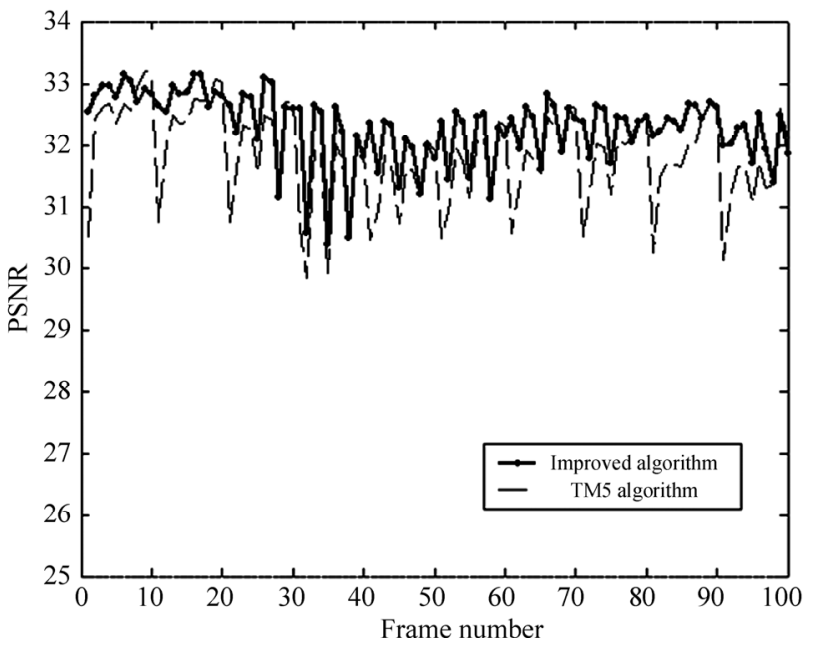

(a) 1.2Mbps

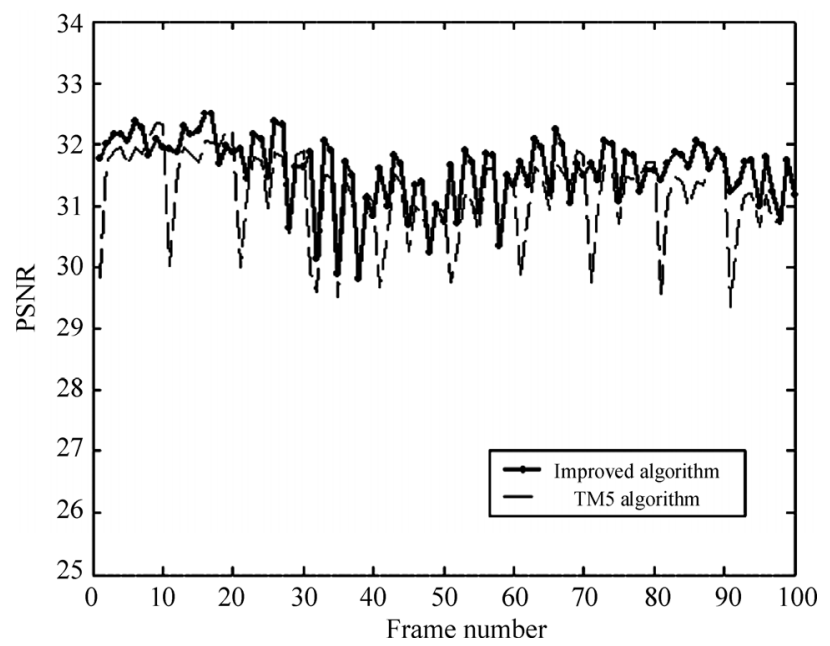

(b) 1.0Mbps

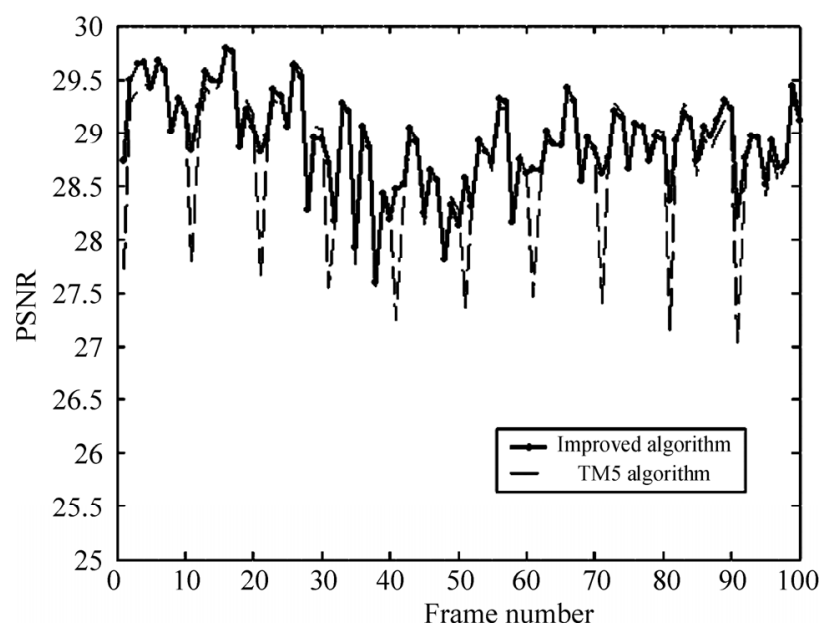

(c) $0.6 \mathrm{Mbps}$

Figure 7. Image qualities of the Train sequence at different bit rates. 
well as simulation experiments. Two indexes are investigated, algorithm complexity and rate-control precision.

In TM5 algorithm, the final selection of the quantification parameter should consider the MB's spatial activity. The spatial activity of the $j$ th MB can be calculated by

$$
a c t_{j}=1+\min \left(v b l k_{1}, v b l k_{2}, \ldots, v b l k_{8}\right)
$$

where

$$
\begin{gathered}
\text { vblk }_{n}=\frac{1}{64} \times \sum_{k=1}^{64}\left(P_{k}^{n}-P_{-} \text {mean }_{n}\right)^{2} \\
P_{-} \text {mean }_{n}=\frac{1}{64} \times \sum_{k-1}^{64} P_{k}^{n}
\end{gathered}
$$

$P_{k}$ are the sample values in the $n$-th original $8 * 8$ block.

Based on above analysis, the complexity of TM5 algorithm is $o\left(n_{1}^{2}\right)$ according to Formula (18). The complexity of proposed novel algorithm is $o\left(n_{2}{ }^{2}\right)$ according to Formulas (8) and (15), where $n_{1}$ is the number of MBs in one frame and $n_{2}$ is the number of available QPs. Since $n_{1}>>n_{2}$, one can see that the complexity of the improved algorithm reduces greatly compared with the original TM5 algorithm.

To further evaluate the overall performance of the proposed algorithm, simulations are conducted on some standard sequences including Alex, Train and so on. We mainly investigate the rate-control precision and image quality fluctuation. Here, image quality is computed by PSNR and rate-control precision is defined as:

$$
\delta=\operatorname{sqrt}\left[\left(R-R^{\prime}\right)^{2}\right] / R
$$

where $R$ is the target number of bits of GOP and $R^{\prime}$ is the actual generated number of bits.

Figures 4-7 give partial experimental results, where Figure 4 and Figure 5 are the results of rate-control precision of TM5 algorithm and the improved algorithm at different bit rates; Figure 6 and Figure 7 are the results of image quality of the two algorithms. From the experimental results, it can be seen that, compared with the TM5 algorithm, the proposed algorithm can both improve the rate-control precision and reduce the image quality fluctuation.

\section{Conclusions and Future Work}

The paper proposed a novel rate-control algorithm based on the TM5 framework of MPEG-2. The drawback of the target bit allocation method of the original TM5 algorithm is improved and a new rate-distortion model is incorporated. The MB-level rate control is adapted to be frame level. As a result, compared with the original TM5 algorithm, the improved novel algorithm not only can enhance the rate-control precision but also can reduce the complexity and the fluctuation of decoded image quality. In our future work, the HVS features will be analyzed and is to incorporate into our rate control algorithm to further enhance the subjective visual quality of coded videos.

\section{Acknowledgments}

This work was supported in part by the Natural Science Foundation of Zhejiang Province (No.Y107740); the Open Project Foundation of Ningbo Key Laboratory (No.2007A22002); the Natural Science Foundation of Ningbo (No.2008A610015).

\section{References}

[1] M. Loren and V. Rahul, "Improved rate control and motion estimation for H.264 encoder,” Proceedings of IEEE International Conference on Image Processing, pp. 309312, 2007.

[2] Z. Z. Chen and K. N. Ngan, "Recent advances in rate control for video coding," Signal Processing: Image Communication, pp. 19-38, 2007.

[3] P. F. Zhao, J. W. Liu, and Q. Li, “A rate control idea and algorithm realization for H.264/AVC," Computer Engineering, pp. 233-249, 2006.

[4] K. Nejat, A. Yucel, and M. M. Russell, "Frame bit allocation for the H.264/AVC video coder via cauchy-density-based rate and distortion models,” IEEE Transactions on Circuits and Systems for Video Technology, pp. 994-1006, 2005.

[5] P. Papadimitriou and V. Tsaoussidis, "A rate control scheme for adaptive video streaming over the internet," [Online], http://www.comp.lancs.ac.uk/ pap- adimp/icc07.pdf.

[6] L. Xu, W. Gao, X. Y. Ji, and D. B. Zhao, "Rate control for hierarchical B-picture coding with scaling-factors," [Online], http://idm.pku.edu.cn/lunwen/2007_2008619101121.pdf.

[7] H. Li and Z. Z. Fu, "Improvement research based on H.264 TM5 rate control algorithm,” Journal of Computer Applications, pp. 749-751, 2007.

[8] T. Y. Tian, "Research and applications of rate control in video coding," Journal of University of Electronic Science and Technology of China, pp. 24-32, 2006.

[9] W. Yuan, "Research for the ratio control algorithm in H.264,” Ph.D thesis, Hefei University of Technology, pp. 9-32, 2006.

[10] H. J. Bi, New Generation Video Compression Coding Standard H.264, Posts and Telecommunications Press, pp. 114-117, 2005.

[11] Z. J. Zhu, F. Liang, G. Y. Jiang, and M. Yu, "Bit-allocation and rate-control algorithm for stereo video coding," Journal on Communications, pp. 15-19, 2007. 